\title{
Artículos
}

\section{Producción discursiva de las políticas de áreas verdes urbanas: Libro Blanco Reverdece tu ciudad y gestión de áreas verdes (2006-2012)}

\section{Discursive production of urban green area policies: White Paper Green your city and green area management (2006-2012)}

María Luisa Ballinas Aquino*

José Álvaro Hernández Flores**

\begin{abstract}
Resumen
El presente artículo desarrolla una propuesta teórico-metodológica que integra diversas estrategias del análisis del discurso con el fin de entender el proceso de la política pública en términos discursivos. Con base en dicho planteamiento, se analiza la producción de políticas de áreas verdes urbanas implementadas en la Ciudad de México durante el periodo 2006-2012. El caso analizado muestra cómo el acercamiento interdisciplinar e interescalar favorece la comprensión de los discursos urbanos y ambientales en su complejidad, evidenciando los mecanismos a partir de los cuales se construyen los conceptos, categorías y narrativas que dotan de legitimidad a la acción pública.
\end{abstract}

Palabras clave: análisis del discurso; áreas verdes urbanas; políticas ambientales; políticas urbanas; Ciudad de México.

* Centro de Investigación y Promoción Cultural para la Integración Comunitaria "Comparte Vida”, A.C. Dirección: 7a. Poniente Norte 565, CP 29000, Tuxtla Gutiérrez, Chiapas, México. Correo: mballinas@colmex.com ORCID https://orcid.org/0000-0002-7381-2363

** El Colegio de México, A.C. Dirección: Picacho Ajusco 20, Ampliación Fuentes del Pedregal, 14110, Tlalpan, Ciudad de México, México. Correo: jalvaro@colmex.mx ORCID: http://orcid.org/0000-0002-8349-0505 


\begin{abstract}
This article develops a theoretical-methodological proposal that incorporates various discourse analysis strategies to explain the public policy process in discursive terms. Based on this approach, the production of urban green area policies implemented in Mexico City during the period 2006-2012 is examined. The case analyzed shows how the interdisciplinary and interscale approach enhances the understanding of urban and environmental discourses in their complexity, evidencing the mechanisms from which the concepts, categories and narratives that give legitimacy to public action are constructed.
\end{abstract}

Keywords: discourse analysis; urban green areas; environmental policies; urban policies; Mexico City.

\title{
Introducción
}

La comprensión de las políticas públicas puede realizarse desde distintas perspectivas analíticas, cada una de las cuales conlleva implicaciones con respecto a la forma en que se concibe e interpreta el proceso analizado. El posicionamiento desde una perspectiva en particular supone no sólo la elección de una concepción sobre el proceso de las políticas públicas, sino también de un conjunto de herramientas analíticas apropiadas para su abordaje teórico metodológico (Mühlhausler y Peace, 2006).

A continuación, se presenta un estudio de la política pública desde una perspectiva discursiva específica, en la cual, mediante el análisis de conceptos, categorías y narrativas, se pretende la comprensión de lenguajes vinculados a: a) el objeto de estudio (áreas verdes urbanas), b) los instrumentos de política pública (normativos y programáticos), y c) los contextos socioeconómicos y políticos que hacen posible la interpretación de la política pública.

En este artículo se desarrolla una propuesta metodológica para identificar y situar en la red de intertextualidades e intratextualidades aquellos conceptos, categorías y narrativas que permiten la conexión entre diversas escalas espaciales y temporales para la producción y reproducción discursiva de las políticas de áreas verdes urbanas. Para ello se analiza un instrumento de política elaborado durante el sexenio de 2006-2012 en el Gobierno del Distrito Federal (hoy Ciudad de México): el Libro Blanco Reverdece tu ciudad y gestión de áreas verdes urbanas. ${ }^{1}$

${ }^{1}$ En el contexto europeo, los "libros blancos" se elaboran con el objetivo de difundir información para poner en marcha un debate y establecer consensos políticos con los diferentes actores sociales tanto gubernamentales como no gubernamentales. En México, la Secretaría de 
Una particularidad de este tipo de estudios es la conformación de un corpus integrado por textos de distinta índole (normativos, programáticos, académicos, etc.) que, pese a sus diferentes formatos y registros estilísticos, mantienen una fuerte asociación derivada de la importancia estructural y funcional que cada uno de ellos tiene en la construcción de una política pública específica. Al considerar en conjunto la política de áreas verdes urbanas, en el corpus desarrollado para este trabajo los textos conforman una red que fundamenta, justifica y hace posible la acción pública en torno al tema analizado, aunque cada uno tiene su propia estructura y sentido.

El presente trabajo se inscribe en el análisis crítico del discurso, ${ }^{2}$ el cual busca contribuir a la discusión tanto del discurso medioambiental en general, como de sus aspectos normativos, en los cuales se observa la vinculación entre las reformas realizadas durante el período de estudio y los intereses de los diseñadores de políticas públicas. De esta forma, el trabajo comparte planteamientos del derecho ambiental, el cual ha sido poco estudiado desde este tipo de perspectiva analítica, tal como afirma Gellers (2015, p. 482):

Hay poca investigación sobre el discurso ambiental que se centra específicamente en el derecho ambiental, y los pocos esfuerzos en los que el análisis crítico del discurso (CDA) ha sido aplicado al estudio del derecho ambiental no han utilizado adecuadamente toda la gama de capacidades analíticas incorporadas a este método.

En este estudio, la inclusión de textos normativos en el análisis discursivo de la política pública resulta indispensable, puesto que "el derecho es fuente de lenguaje en la sociedad y el derecho es un espacio para la expresión de poder" (Gellers, 2015, p. 484).

Para el análisis del discurso, los conceptos -como el de áreas verdes urbanas-no sólo se presentan como conocimientos comunicables, sino que en sí mismos son medios para la difusión de ideas dominantes ${ }^{3}$ que señalan la

la Función Pública los define como "el documento público gubernamental en el que se hacen constar las acciones y los resultados obtenidos más destacados de un programa, proyecto o asunto relevante y trascendente de la Administración Pública Federal" (artículo 2, fracción II). En ellos se describen, en términos conceptuales, las acciones legales, presupuestarias, administrativas, operativas y de seguimiento que se hayan realizado, así como los resultados obtenidos del programa, proyecto o asunto de que se trate (Ballinas, 2018).

${ }^{2}$ El análisis crítico del discurso entiende el discurso en situación y en contextos amplios, dentro de "procesos de comercialización del discurso público" (Fairclough, 2008, p. 177).

${ }^{3}$ Gellers (2015, p. 484) observa las luchas de poder que están presentes en las políticas ambientales y la importancia de las instituciones capaces de afirmar el poder cuando se transforman reglas y normas. 
dirección que tendrán que seguir (en términos de "bondad") las acciones de política pública. De este modo, el estudio de los conceptos movilizados en las políticas específicas constituye una posibilidad de acercamiento al análisis de la producción discursiva desde un posicionamiento crítico, a partir de la premisa de que es posible reconocer en el discurso las voces hegemónicas que se imponen de manera sutil, sin el uso de la fuerza, ${ }^{4}$ mediante la persuasión y la negociación. En este sentido, el presente trabajo pretende identificar, por un lado, cuáles son las formas de movilización discursiva de los conceptos, en diversas escalas, para convertirse en fundamento de políticas públicas específicas; y por otro, cómo se significan los conceptos en el marco de las categorías (macroconceptos) y narrativas provenientes de las diversas disciplinas que convergen en los textos de políticas públicas.

Para el estudio de los conceptos dentro del análisis discursivo de las políticas públicas, se propone el siguiente itinerario: 1) el posicionamiento teórico sobre las construcciones conceptuales y narrativas; 2) los conocimientos y lenguajes disciplinares en el discurso de las áreas verdes urbanas; 3) los lenguajes disciplinares en el mundo global; 4) el concepto de áreas verdes urbanas en relación con otros conceptos; 5) el concepto de áreas verdes urbanas en relación a otras categorías (macroconceptos), y 6) las narrativas recurrentes en las políticas de áreas verdes urbanas.

En este recorrido es posible entretejer los aportes de las diversas disciplinas al uso del lenguaje de la política específica, la influencia de la globalización en la construcción y la difusión de los conceptos en los documentos de políticas públicas que traspasan las escalas, y el reconocimiento de los campos temáticos que tienen una función contextual.

Los ejemplos empíricos que se presentan a lo largo del trabajo hacen referencia al texto núcleo del análisis, el Libro Blanco Reverdece tu ciudad $y$ gestión de áreas verdes, ${ }^{5}$ elaborado por la Secretaría del Medio Ambiente de la Ciudad de México durante la gestión 2006-2012, así como a un conjunto de documentos producidos en diversas escalas espaciales y temporales, los cuales tienen una relación directa con el texto núcleo del estudio. Aunque dichos documentos pertenecen a diversos géneros y poseen marcos interpretativos propios, en esta investigación no se hace una discusión ex-

${ }^{4}$ De acuerdo con la propuesta de Conde (2017, p. 22), quien distingue entre la dominación y la hegemonía, la primera constituye una imposición, mientras que la segunda se refiere a negociaciones que fortalecen liderazgos, en donde intervienen posiciones geográficas, económicas y políticas.

${ }^{5}$ Para observar el análisis realizado con mayor amplitud en cuanto a los textos incluidos, es necesario acercarse a la tesis Análisis del proceso discursivo de la política de áreas verdes urbanas en la Ciudad de México 2006-2012, sustentada por María Luisa Ballinas Aquino en El Colegio de México, A.C. 
haustiva de cada uno de ellos, sino que se considera el texto exclusivamente con relación a su aporte a la construcción de conceptos, macroconceptos (categorías) $^{6}$ y narrativas vinculadas con el concepto central del instrumento de política pública analizado: las áreas verdes urbanas.

\section{Posicionamiento teórico-metodológico sobre las construcciones conceptuales}

Para fines del presente trabajo se propone una construcción del concepto áreas verdes urbanas con base en las relaciones intra, inter y supraconceptuales. ${ }^{7}$ De manera análoga (aunque en una escala diferente), cuando se habla del análisis del discurso se retoman los planteamientos de Van Djik, quien considera "que las oraciones no deben estudiarse aisladamente, sino en relación a las demás oraciones de un discurso" (Van Djik, 1991, p. 58).

Desde este tipo de enfoque se considera que el concepto se construye en la interacción entre lo material y lo inmaterial, en un proceso social dinámico que encuentra su definición de límites (los cuales se flexibilizan o se hacen rígidos) en la interacción con otros campos conceptuales, dependiendo de las dinámicas del proceso social que se manifiesta en el lenguaje. En este sentido, Koselleck (2003) afirma que el concepto tiene una dimensión histórica, que implica tanto factores de cambio social como acumulación de experiencias, por lo cual se puede afirmar que el concepto permite reconocer la experiencia de las políticas públicas expresadas en el tiempo y en el espacio, lo cual resulta una consideración fundamental para este trabajo.

La construcción del concepto está sujeta al devenir histórico que hace posible las transformaciones en cuanto a sus significados y a las formas de expresión lingüística. Dichos cambios se deben a las variaciones socioculturales que se constituyen en marcos referenciales y que tienen implicacio-

${ }^{6}$ La diferenciación entre concepto y categoría en este texto es distinta de la realizada por Koselleck (2003), para quien el concepto está ligado más a las fuentes históricas, mientras que las categorías se entienden como elaboraciones académicas. En este caso, la distinción se realiza en torno al nivel de concreción que tiene el término: si hace referencia a objetos más concretos, se considera un concepto; mientras que, si se alude a aspectos más generales, con mayor dificultad de aprehensión, se hace referencia a una categoría.

${ }^{7}$ La relación intraconceptual se refiere a las variantes en cuanto a las significaciones que se encuentran vinculadas a un término determinado. Cuando se alude a lo interconceptual, se hace referencia a los diferentes términos que en cierto modo pueden ser intercambiables, dado que comparten algunos aspectos relacionados con sus significaciones. Finalmente, lo supraconceptual alude a la relación del concepto con aquellos macroconceptos (categorías) que brindan un espacio de significación al concepto, el cual se entiende y discute de forma distinta cuando se moviliza vinculado a una categoría o a otra (Ballinas, 2018). 
nes en las formas de vinculación del concepto consigo mismo (elementos que lo integran y sus tipologías), así como con otros conceptos, categorías (macroconceptos) y narrativas en las que son movilizados.

Desde la perspectiva de Bourdieu (2008), el concepto contribuye a producir la llamada realidad objetiva, mediante la construcción de una representación mental en la cual los agentes manifiestan sus intereses e intenciones. De esta forma, se plantea una forma de analizar las diversas propiedades atribuidas al concepto de áreas verdes urbanas, en donde lo simbólico se constituye como una representación, entendida desde la perspectiva de Bourdieu como un "enunciado performativo que pretende hacer realidad lo que enuncia” (Bourdieu, 2008, p. 117).

En cuanto a la conformación simbólica de las áreas verdes urbanas, en este trabajo se discuten los niveles de relación observados en las construcciones conceptuales, con las regularidades y discontinuidades asociadas a la comprensión de dicho concepto. Las áreas verdes constituyen un concepto en el cual convergen conocimientos diversos sobre dicho espacio, y cuya conformación es posible mediante las diversas formas de vinculación entre el concepto central y otros conceptos con los cuales se encuentra en un espacio de intersección nocional.

En resumen, para fines de la investigación realizada, el concepto se construye a partir de la relación intra, inter y supraconceptual o categorial, mediante marcos referenciales tanto del concepto central como de los asociados en el análisis.

Para el análisis de la política de áreas verdes urbanas se tomaron en consideración tres tipos de construcciones discursivas: conceptos, macroconceptos o categorías y narrativas.

Dado que el concepto central sólo se construye en relación a otros conceptos, el acercamiento al concepto de áreas verdes urbanas en el instrumento de política pública analizado se realiza a partir del análisis de las relaciones del concepto central con otros conceptos que contribuyen a establecer los alcances y límites de sus significaciones.

En este estudio se considera que los conceptos movilizados en los discursos de las políticas públicas se construyen en diálogo constante con las diversas disciplinas, algunas veces para justificar políticas, mientras que, en otros casos, los conceptos surgidos en la praxis del lenguaje de las políticas se revisten de significado a partir de las discusiones disciplinares, por lo que resulta ineludible el vínculo entre los conceptos y los lenguajes disciplinares (Ballinas, 2018).

Por ejemplo, la Ley Ambiental del Distrito Federal (hoy Ciudad de México) define al área verde como "toda superficie cubierta de vegetación, 
natural o inducida que se localice en el Distrito Federal", mientras que otros documentos internacionales se refieren a dichos espacios urbanos desde una perspectiva económica, como un área de oportunidad para mejorar la calidad de vida (Ballinas, 2018). Por otra parte, en el Libro Blanco Reverdece tu ciudad y gestión de áreas verdes (Sedema, 2012a) se mencionan conflictos relevantes relacionados con dichos espacios, en los cuales se consideran aspectos biológicos (diversidad biológica y plagas), urbanos (planeación y diseño de las ciudades), sociales (como espacios para la interacción, pero también para el vandalismo) y de mantenimiento (acciones de gobierno para el cuidado de estos lugares públicos).

\section{Esquema 1}

\section{Construcción del concepto central}

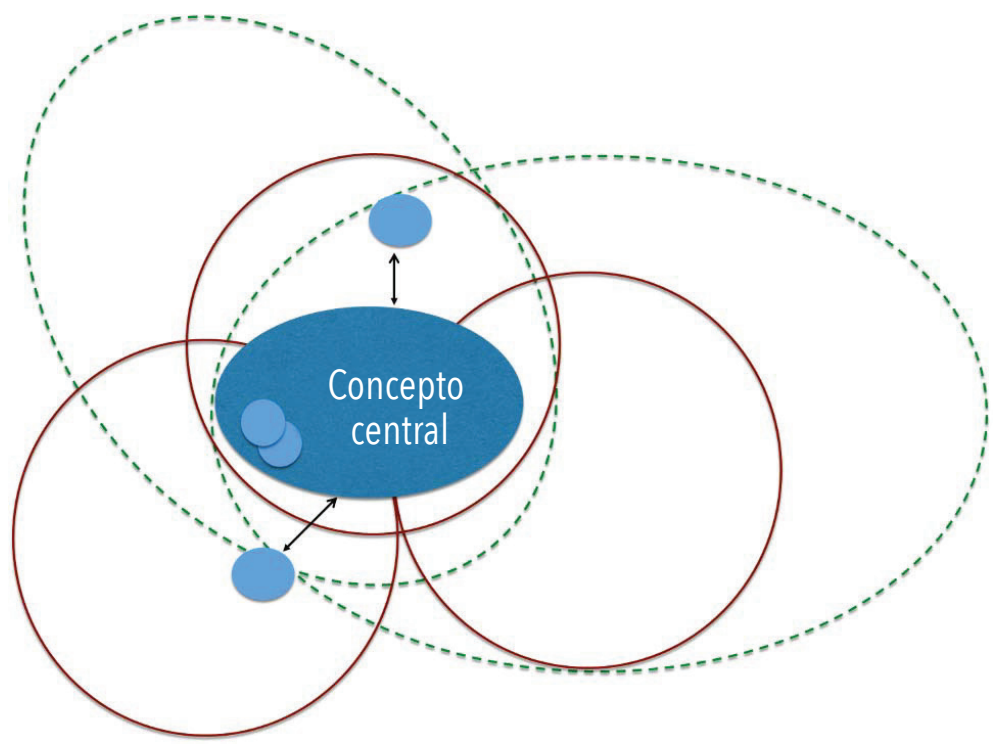

Nota: Las elipses punteadas representan a las narrativas; los círculos, a las categorías; y los puntos, a los conceptos. El concepto central se significa en función de las clasificaciones internas, de los conceptos cercanos y de los marcos interpretativos que aportan las categorías y narrativas en las cuales se movilizan.

Fuente: Elaboración propia. 
Al hacer referencia a los macroconceptos o categorías, se consideran términos que, a diferencia de lo que aquí se denomina conceptos, no se refieren a un objeto concreto. Por ejemplo, las áreas verdes urbanas se consideran un concepto, puesto que hacen referencia a un espacio concreto con características específicas; sin embargo, el término de sustentabilidad, siendo más amplio, no se refiere a un objeto determinado, sino a un conjunto de acciones y significaciones que pueden discutirse. En este sentido, las categorías pueden pertenecer a campos de estudio que integran muchas disciplinas, tienen mayor complejidad y en algunos casos pueden coincidir con lo que Molle (2008) menciona sobre aquellos conceptos que emergen como ideales, a los cuales no es posible llegar pero que se convierten en el "motor" para poner en movimiento ideas y acciones. Por ejemplo, cuando se habla de los problemas relacionados con las áreas verdes urbanas, en el Libro Blanco analizado se menciona la "calidad" de los servicios ambientales y sus "beneficios sociales" (Sedema, 2012a, p. 7), términos amplios que pueden tener comprensiones diversas y, por tanto, dar lugar a ciertas ambigüedades. Sucede lo mismo con la asociación de las áreas verdes urbanas al tema de la "participación ciudadana" o de la "calidad de vida" (Sedema, 2012a, p. 7), vinculación discursiva que coloca a dichos espacios en medio del proceso social, no sólo como espacios que hay que cuidar, sino también como áreas que, al ser conservadas, producen beneficios en la vida de las personas.

En este estudio, las narrativas se analizan principalmente a través de la observación de las formas de comprensión del tiempo y el espacio en los programas vinculados con las áreas verdes, así como a través del análisis de los puentes discursivos y de las tríadas de conceptos y/o categorías.

Mediante los puentes discursivos se construyen narrativas que relacionan conceptos o categorías, con énfasis en el punto de partida y el de llegada, pero incorporando un concepto o categoría intermedia que tiene gran relevancia en términos discursivos porque brinda la posibilidad de insertar la narrativa en un tema en particular, aunque el punto de partida y el de llegada no tengan relación directa con dicho tema (Ballinas, 2018). Por ejemplo, cuando se dice que la "participación ciudadana en el manejo de las áreas verdes incide en la democratización de las sociedades", el punto de partida es la "participación ciudadana", mientras que el punto de llegada es la "democratización de las sociedades". En este caso, se podría prescindir de la narrativa del manejo de las áreas verdes; sin embargo, si ésta se quiere colocar en el marco de un texto ambiental, dichos puentes pueden resultar de gran valor discursivo. 
Las tríadas, por su parte, son asociaciones que se encuentran de manera recurrente entre determinados conceptos y categorías. Por ejemplo, la tríada "pobreza- presión sobre los recursos- deterioro ambiental" es común en los temas ambientales. Estas tríadas fortalecen narrativas que pueden llegar a tener una presencia generalizada en diversos textos, transformándose en emblemas discursivos que se movilizan para conseguir los fines discursivos de la política pública.

Esta propuesta teórico-metodológica considera la multirrelacionalidad y la multidimensionalidad de los conceptos, con lo cual se pretende el análisis de las significaciones tanto del concepto central como de las categorías y narrativas, así como el reconocimiento de algunas estrategias discursivas utilizadas en las políticas públicas que utilizan el concepto como medio para fines diversos, no necesariamente relacionados con el concepto central, mientras que incorporan ambigüedades que se reproducen en diversas escalas espaciales, temporales y temáticas.

\section{Conocimientos y lenguajes disciplinares en el discurso de las áreas verdes urbanas}

En el Libro Blanco Reverdece tu ciudad y gestión de áreas verdes (Sedema, 2012a) convergen tres tipos de lenguajes disciplinares: el biológico, el económico y el normativo. El primero atiende más a cuestiones técnicas, el segundo emerge en muchos casos como requisito y fin de la política específica, mientras que el último establece el espacio posible para la acción pública.

El lenguaje de las ciencias biológicas está presente con la finalidad de fundamentar las acciones en los conocimientos con los cuales se brindan certezas a quienes leen el texto y a quienes tienen a su cargo la ejecución de las acciones de política pública. La función del uso del lenguaje biológico no es explicativa, puesto que no se pretende una comprensión de las implicaciones que tienen los conocimientos que se movilizan, sino que están puestas en el documento para provocar la confianza en las autoridades, que "saben lo que hacen". Algunas narrativas encontradas en textos de política de áreas verdes urbanas que utilizan el lenguaje biológico hacen referencia a las condiciones de desarrollo del arbolado, a las plantas parasitarias que afectan a las diversas familias botánicas presentes en la ciudad y a las deficiencias en la planeación de los espacios verdes (al no considerar aspectos de diseño, técnicos y de conocimiento de la biología). Las dinámicas biológicas van de lo descriptivo a lo funcional en el marco del conocimiento 
aplicado, aunque sin profundizar en las dinámicas ecosistémicas en las que se encuentran inmersas las áreas verdes.

En el Libro Blanco referido, el lenguaje económico emerge con las estrategias que se relacionan con las acciones de cuidado y protección del medio ambiente mediante las propuestas presentadas. Desde la perspectiva económica, se reconocen estrategias de política pública como los incentivos fiscales, los financiamientos con tasas preferenciales, las inversiones, la rentabilidad de los proyectos, así como las estrategias en sentido negativo cuando se infringe la ley. Cada uno de estos términos se inserta en la dinámica del mercado de una forma particular, por lo cual se requiere un conocimiento en el área económica con diversos grados de especialización, dependiendo del desarrollo de la política en la que se encuentre, puesto que no es lo mismo diseñar los instrumentos fiscales asociados al programa, que realizar la gestión empresarial (persona moral) o ciudadana (persona física) ante las acciones implementadas. Las áreas verdes urbanas se encuentran asociadas al incremento del valor del suelo y de la vivienda circundante, con lo cual se considera un aspecto positivo para la economía.

Por otra parte, el lenguaje normativo tiene especial importancia en la construcción discursiva de las políticas debido a que pertenece al ámbito de la fundamentación de las acciones públicas que se tienen que presentar con la mayor inteligibilidad. Por ello, el lenguaje normativo que se presenta en el Libro Blanco aludido se refiere a las interacciones entre los actores sociales, en términos de corresponsabilidad y competencias; asimismo, se considera el fortalecimiento del marco legal y normativo mediante la actualización de las normas que se aplican al tema de las áreas verdes urbanas en la Ciudad de México. Finalmente, el Libro Blanco Reverdece tu ciudad y gestión de áreas verdes integra diversos programas, acciones y proyectos en marcos normativos que convergen en el tema de las áreas verdes urbanas.

Los lenguajes disciplinares en las políticas de áreas verdes urbanas pueden resumirse en el Esquema 2. Dichos lenguajes permiten identificar la relación que tendrán los diversos actores sociales con respecto a las áreas verdes urbanas. Por ejemplo, al fundamentar con el lenguaje biológico la acción pública, se subraya la importancia que adquiere la presencia del biólogo como actor social. Al mismo tiempo, el lenguaje permite observar cómo van adquiriendo relevancia otros actores sociales, tales como los economistas, relacionados con las propuestas de proyectos de incentivos fiscales y el incremento en el valor de las viviendas cercanas a las áreas verdes.

En estos lenguajes emerge el término de "necesidades", presente en la lucha discursiva entre la gestión urbana en general y las acciones ambien- 
tales concretas, con lo cual se pueden identificar formas de entender al ser humano con relación a su entorno y las implicaciones que ello tiene en la política de áreas verdes urbanas. Por ejemplo, en el Libro Blanco analizado se consideran las necesidades de vivienda, servicios y vialidades, vinculadas a las áreas verdes en las ciudades (Sedema, 2012a, p. 27); asimismo, los instrumentos de políticas de áreas verdes urbanas se presentan como una necesidad (p. 13). Finalmente, aunque en los tres tipos de lenguaje se hace alusión a las "necesidades", las formas de comprensión están diferenciadas en cuanto a la pregunta ¿necesidades de qué?, mientras que en todos los casos se establece una comprensión vinculada a los requerimientos de satisfactores que movilizan a la acción pública, a manera de utopía que implica la realización y, al mismo tiempo, la inaccesibilidad.

\section{Esquema 2}

Tipos de lenguajes disciplinares en las políticas de áreas verdes urbanas

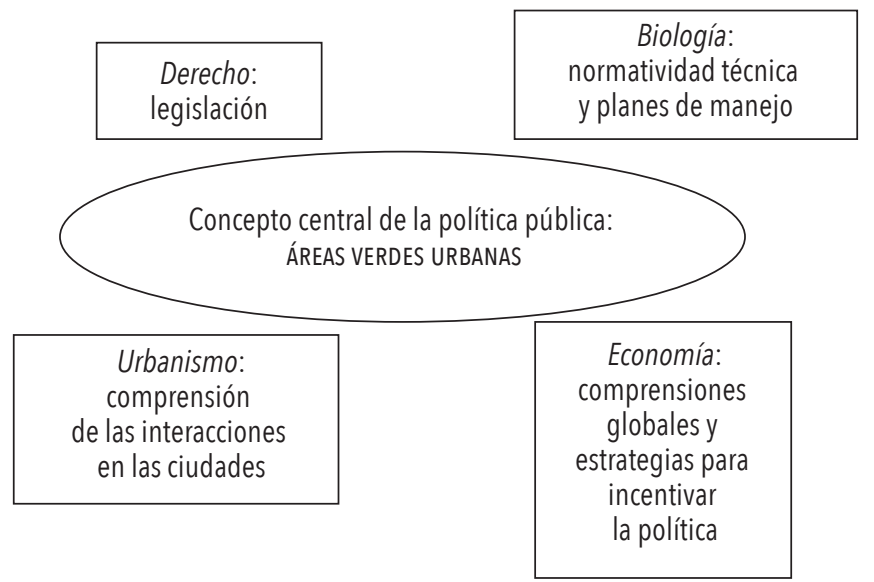

Fuente: Elaboración propia.

\section{Los lenguajes disciplinares en un mundo global}

En este apartado se pondrá atención principalmente a los lenguajes disciplinares de la economía y de la biología en las construcciones interescalares que se relacionan con la comprensión de las áreas verdes y la acción pública 
en torno a dichos espacios. Asimismo, se identifica el tema de la "necesidad" como un dispositivo discursivo vinculado a ambos tipos de lenguaje.

Desde una perspectiva global, las áreas verdes se visualizan como necesarias para mantener la calidad de vida porque a ella se asocian diversos efectos positivos contrapuestos a los efectos contaminantes vinculados a la urbanización. Se observa, por lo tanto, que la relación entre lo ambiental y lo urbano permite enfatizar la emergencia de "necesidades" y, en algunos casos, favorece la dinámica de consumo que incide en los mercados "verdes", los cuales tienen productos "a la medida" de las necesidades. La presencia de actores sociales, como el Banco Interamericano de Desarrollo (BID), permite identificar financiamientos en torno a los espacios verdes, como se afirma en el Seminario de Áreas Verdes en Latinoamérica y el Caribe (1998, p. 4): "En la Ciudad de México está en marcha un importante proyecto de enverdecimiento urbano que cuenta con el auspicio parcial del BID, el cual puede servir como un ejemplo para otros proyectos similares en la región". En este sentido, aparece la construcción de una política mediante la configuración de un problema que tiene una solución (Aguilar, 2003), en la cual se subraya la presencia de productos disponibles. Aun cuando las soluciones propuestas se establecen con base en productos del mercado, la acción pública no siempre logra resolver los problemas públicos en los que convergen aspectos relacionados con las interacciones entre los diversos actores sociales y las formas de comprender los alcances, límites y niveles de solución que es posible atender con los productos del mercado "verde".

Los lenguajes disciplinares permiten el fortalecimiento de las construcciones globales tanto de los problemas ambientales como de las soluciones propuestas, debido a que establecen "necesidades" en todos los ámbitos de la vida, bajo los principios del mercado, e inciden en la construcción de las políticas, las cuales surgen en medio de la no regulación del mercado, pero con dependencia y sometimiento a una serie de regulaciones positivas nacionales y consuetudinarias internacionales. Por lo tanto, se puede afirmar que los lenguajes disciplinares en torno a una política pública, cuando se plantean desde una perspectiva global, atienden a la configuración de necesidades, en un contexto neoliberal que impone al Estado tanto la liberación del mercado como la apertura a espacios de inversión (Durand, 2014). Al mismo tiempo, se apuesta por un proceso sociocultural que permita la naturalización del mercado "como el medio para evaluar y distribuir las necesidades y los lujos de la vida" (March, 2013, p. 129). Así se ha propuesto desde las comprensiones del desarrollo promovidas, entre otras, por las Cumbres de la Tierra, como la de Río de Janeiro (CNUMAD, 1992), cuando a partir del concepto de intergeneracionalidad, se justificó el derecho al 
desarrollo con la finalidad de "responder equitativamente a las necesidades de desarrollo y ambientales de las generaciones presentes y futuras" (principio 3).

En este sentido, la globalización, mediante el concepto de "necesidades", ha logrado movilizar los lenguajes de las diversas disciplinas que inciden en la construcción de las políticas públicas específicas, y más aún, dicho concepto (entre otros), permite dinamizar su proceso discursivo. Por ejemplo, en el Libro Blanco estudiado, mediante el lenguaje de la biología se justifica la "necesidad de elaborar un inventario de áreas verdes y la figura jurídica de las áreas de valor ambiental" (Sedema, 2012a, p. 6).

Por otra parte, en el ámbito internacional se observa una tendencia a crear un lenguaje común entre quienes formulan las políticas, los empresarios y la sociedad, para facilitar la visibilización de los problemas ambientales y la incorporación de determinadas ideas (mediante el lenguaje) en la toma de decisiones (Spash y Aslaksen, 2015, p. 249). Con ello, se establece la idea de que, a través del lenguaje común, especialmente de la economía, se va construyendo una realidad para la comprensión del tema ambiental.

\section{Áreas verdes urbanas: la construcción del concepto consigo mismo}

En el Libro Blanco Reverdece tu ciudad y gestión de áreas verdes (Sedema, 2012a), el concepto de áreas verdes no se define mediante el uso del verbo "ser o estar", sino que se construye a partir de los atributos que informan sobre las cualidades de dichos espacios.

En el texto analizado, las áreas verdes tienen una condición (se hace referencia a las "condiciones actuales de las áreas verdes") y una posición (en términos de ubicación y de distribución espacial).

Dos características que permiten mayor especificidad en cuanto al tipo de área verde al que se refiere el texto se encuentran en la distinción entre las áreas verdes arboladas y las no arboladas, así como entre las áreas verdes públicas y las privadas. La primera distinción se basa en un tipo de vegetación que se construye discursivamente como eje de distinción: el árbol; mientras que la segunda distinción se hace con respecto al estatus público o privado del área en cuestión, en donde los actores sociales involucrados tienen responsabilidades diferenciadas.

Entre los actores sociales que juegan un papel importante y cuyos roles se ven transformados por el estatus de las áreas verdes urbanas, se encuentran: los ciudadanos, las autoridades locales, las autoridades federales 
(incluyendo las de aspectos fiscales), y quienes tienen el conocimiento teórico y práctico sobre dichos espacios (profesionales en alguna disciplina afín y jardineros). De esta forma, se observa cómo una diferencia entre las áreas verdes enunciadas tiene implicaciones distintas en la comprensión y en las acciones a partir de las cuales se construyen discursivamente (como acto de habla) dichos espacios, mediante una operación dicotómica donde una de las partes excluye a la otra, estableciendo determinados límites sobre el concepto. Por ejemplo, cuando se hace referencia a las áreas verdes urbanas públicas se consideran acciones que no son aplicables a los espacios privados, y viceversa; como cuando se establece la actividad de cuidado de las áreas verdes realizada por los jardineros que laboran para una instancia gubernamental, quienes realizan la acción exclusivamente en los espacios públicos.

Por otra parte, la construcción del concepto se realiza también mediante estrategias discursivas como el uso de adjetivos calificativos. En este caso, se utiliza el concepto "áreas verdes saludables" para resaltar una característica propia de los seres vivos: la presencia o ausencia de un funcionamiento óptimo en términos físicos y químicos. En el texto se menciona dicha situación haciendo referencia a los problemas de las áreas verdes urbanas. Sin embargo, hay una diferencia importante entre considerar a un área verde enferma y valorar problemas de las áreas verdes, puesto que, dentro de las complejidades propias de los ecosistemas, parece más fácil atender la segunda situación, dado que no se expresa como un atributo intrínseco al área verde, sino como una situación externa al ser vivo en cuestión. Aunque las áreas verdes hacen alusión a un conjunto de interrelaciones entre seres vivos y materia inerte, el calificativo "saludable" acerca el concepto a una comprensión unitaria del ser. El vandalismo y el mal uso de las áreas verdes pueden entenderse como problemas provocados por los seres humanos que tienen problemas en la interacción con y en tales espacios.

Otros adjetivos utilizados son "público" y "privado", lo cual se convierte en una característica imprescindible para determinar el tipo de gestión que es factible dado su estatus legal y social (en cuanto al acceso).

Las acciones realizadas sobre las áreas verdes pueden tener diversos gradientes de control, puesto que cada acción tiene implicaciones distintas en la forma de interactuar con dichos espacios. En el texto analizado, las áreas verdes también se comprenden como un espacio habitado por seres vivos que parecieran estar en un estado de pasividad, sobre el cual se pueden realizar acciones como: atención, cuidado, manejo adecuado, conservación, mantenimiento, construcción, rehabilitación, administra- 
ción, preservación, protección, restauración, forestación, reforestación, fomento, vigilancia, creación, saneamiento, mejoramiento, recuperación, inducción.

Todas estas acciones se pretenden realizar mediante el desarrollo de nuevos mecanismos administrativos y la aplicación de programas. En este sentido, se anota que: "el desarrollo óptimo de las áreas verdes urbanas requiere de continuar con estas acciones y el desarrollo de otras tareas" (Sedema, 2012a, p. 66).

El control ejercido sobre el espacio, y lo que ahí se reproduce, se expresa a través de la insistencia en la elaboración de un inventario de áreas verdes. Las diferencias entre los inventarios que se han realizado marcan una diferencia en los instrumentos de planeación que utilizan dicha información.

Otro elemento en la construcción del concepto de área verde urbana es la tipología (presente en la legislación ambiental y local), que se analiza en el Cuadro 1, ya que nos permite identificar la forma de segmentar dichos espacios mediante la formación de una amalgama de "tipos" que aluden a diversos criterios. Las tipologías se establecen con respecto a criterios que consideran las características de la vegetación, el estatus legal, la distinción de escalas, la conectividad y los beneficios sociales, económicos, escénicos, culturales y ecológicos.

En este caso, se observa la distinción entre áreas naturales protegidas (con competencia federal y local) y la referencia directa a las áreas verdes urbanas. En la legislación local existe una distinción entre áreas verdes urbanas y áreas naturales protegidas, aunque ambas consideran el criterio de la conectividad de las áreas verdes con otros recursos, especialmente con el hídrico. Sólo en el caso de las áreas verdes urbanas se manifiesta la relación de las áreas verdes con el espacio urbano. Esta observación, junto con las adiciones realizadas a la Ley Ambiental del Distrito Federal en el período de estudio (zonas de recarga de mantos acuíferos, áreas de valor ambiental y las demás análogas), permite describir la apertura del tema hacia la conectividad con otros recursos naturales como una característica discursiva que pasa de las fuentes normativas a las construcciones de áreas verdes presentes en el Libro Blanco aquí analizado. 


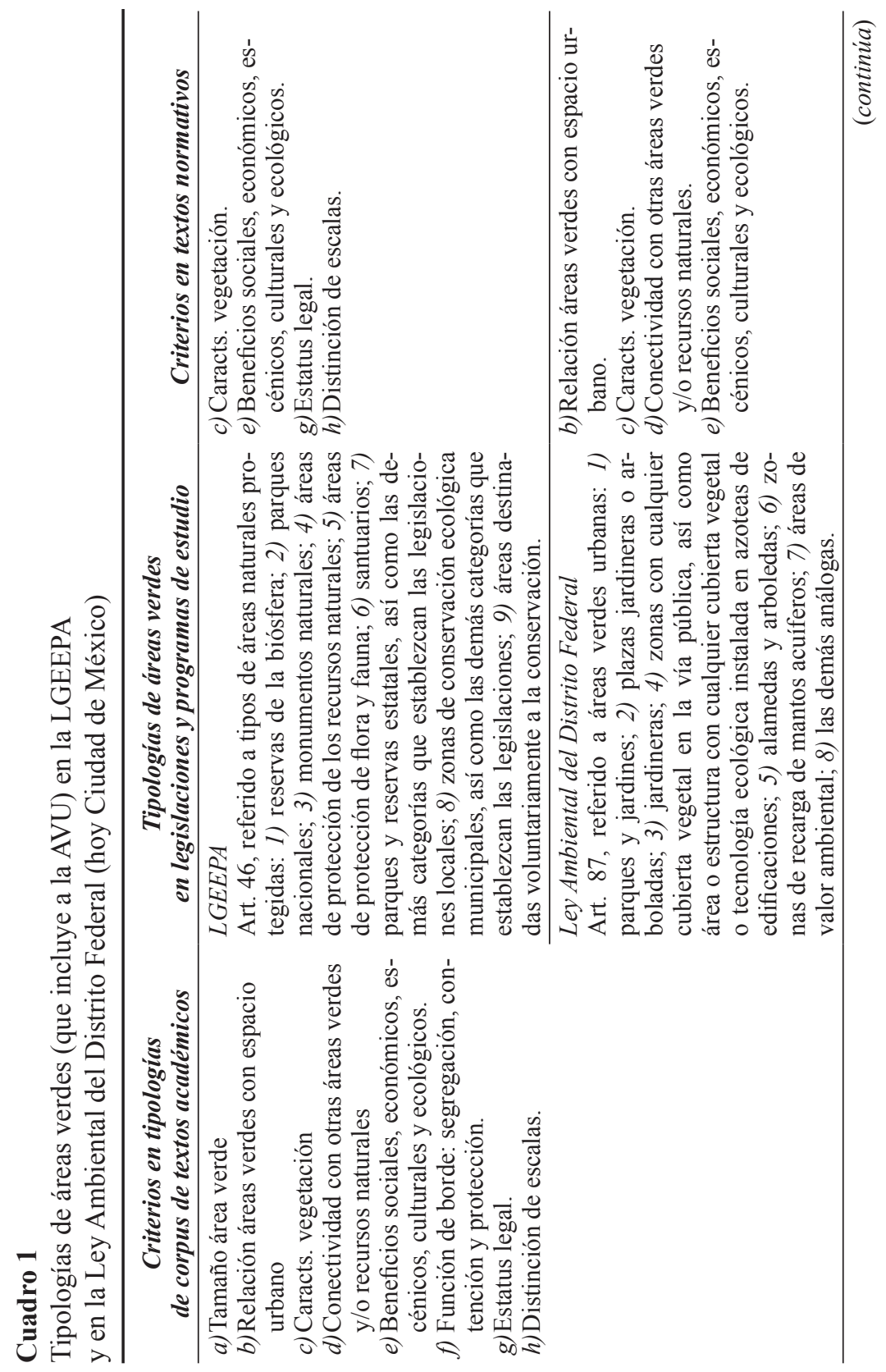




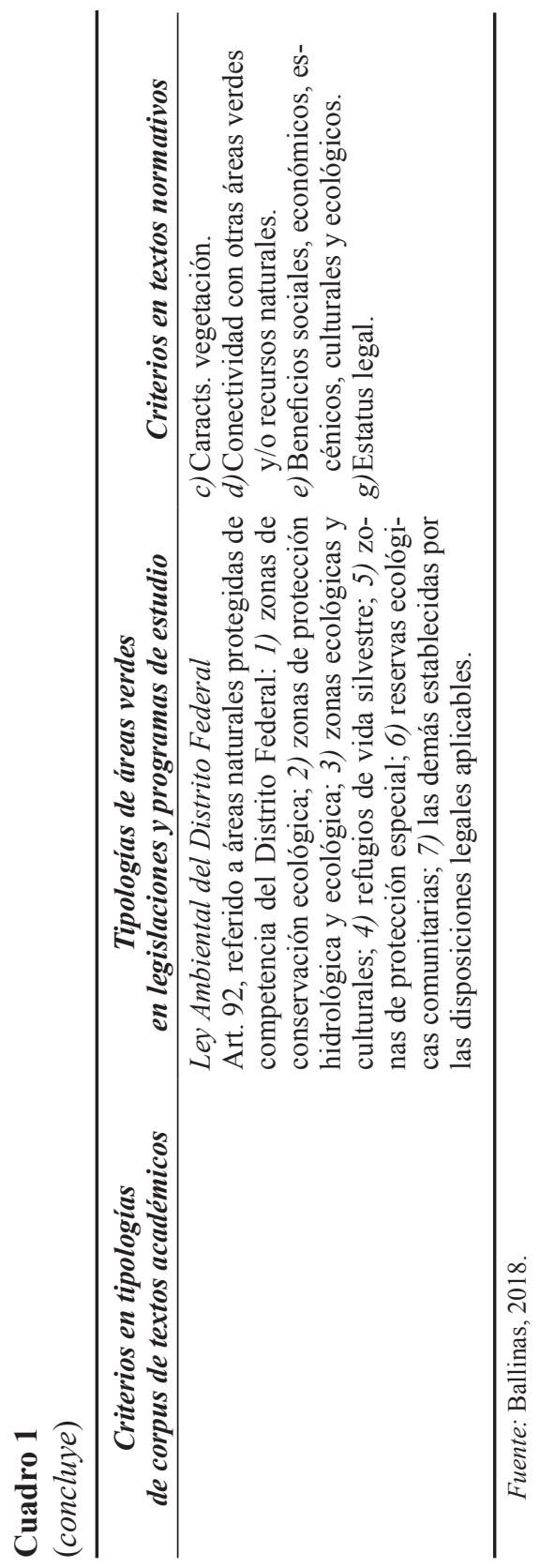




\section{Áreas verdes urbanas: concepto en relación con otros conceptos}

En el Libro Blanco Reverdece tu ciudad y gestión de áreas verdes, además de citar la tipología de la Ley Ambiental del Distrito Federal, se presentan clasificaciones como las siguientes:

Bosques, barrancas, parques, jardines, camellones, glorietas, entre otros [Sedema, 2012a, p. 3].

La clasificación uniforme de las áreas verdes de las 16 delegaciones en las categorías de áreas de valor ambiental, áreas naturales protegidas, escuela y/o dependencia pública, parque (jardín, plaza, deportivo, plazuela), alameda, vialidad (camellones, glorietas, isletas, triángulos, bordos canales), unidad habitacional, panteón, vivero, bosque y barranca [Sedema, 2012a, p. 3].

Se aclara en el texto que se tiene una tipología común entre las 16 delegaciones, y con dicha tipología, una forma dominante de concebir las áreas verdes urbanas, lo cual tiene impacto en la práctica de la política urbana.

A diferencia de las clasificaciones presentes en los documentos más generales, ya sea en cuanto a la temática o en la escala espacial, en esta clasificación se tiene una mayor consideración de las características urbanas en las que se encuentra el área verde. Del mismo modo, cada tipo de área verde tiene características físicas distintas y se vincula no sólo con los espacios urbanos, sino también con funciones diversas. Por ejemplo, en el texto se menciona que:

En los viveros se produce una gran diversidad de especies vegetales (árboles, arbustos y ornamentales) adecuadas a las diferentes condiciones de suelo, clima, infraestructura y mobiliario urbanos [Sedema, 2012a, p. 49].

En las tipologías se observa una distinción entre aquellos espacios verdes que favorecen los aspectos ambientales, tal como sucede con la incluida en la Ley Ambiental del Distrito Federal (como cuando se alude a la zona de recarga de acuíferos), a diferencia de aquellas que enfatizan los aspectos urbanos (por ejemplo, al referirse a la naturación de azoteas), como ocurre en las otras tipologías encontradas en el Libro Blanco Reverdece tu ciudad y gestión de áreas verdes, donde se considera el vínculo de lo verde con lo urbano. Un dato que también se vincula a las tipologías es el de los indicadores, puesto que, dependiendo de la tipología, se realizará la inclusión o exclusión de las áreas verdes en la determinación de un indicador. Por 
ejemplo, si se consideran como áreas verdes únicamente a los espacios arbolados, la cuantificación de áreas verdes urbanas por habitante puede ser mucho menor que si se considera como área verde al espacio que tiene cualquier tipo de vegetación.

La determinación de indicadores facilita el paso de una descripción a una comparación, puesto que se posiciona la cantidad de área verde de una delegación frente a la de otra. La inclusión de indicadores dentro del discurso tiene implicaciones distintas a las de las tipologías debido a que los primeros dan la sensación de objetividad, mientras que las segundas mantienen la delimitación de las clases, pero sin parámetros cuantitativos.

Las observaciones anteriores permiten afirmar que en el discurso de áreas verdes urbanas del Libro Blanco analizado no se hace una distinción explícita entre las áreas verdes a nivel del suelo y en azotea, ni se refieren las diferencias de la experiencia urbano-rural y de la vegetación urbana desde su verticalidad. Sin embargo, existen diferencias que se señalan de forma explícita en otro Libro Blanco que se elaboró en el mismo período de gobierno local: el Programa de Certificación de Edificaciones Sustentables (Sedema, 2012b), en el que se distingue a los actores sociales que se vinculan con cada tipo de área verde (a nivel de suelo y azotea), lo que conduce al desarrollo de estrategias normativas para su implementación.

\section{Áreas verdes urbanas: concepto en relación con categorías (macroconceptos)}

Las categorías presentes en el Libro Blanco Reverdece tu ciudad y gestión de áreas verdes permiten identificar aquellos marcos analíticos donde las áreas verdes tienen alguna participación, brindando un espacio de significado tanto a la categoría y los conceptos asociados, como al propio concepto central (área verde urbana). A continuación, se presentan algunos ejemplos de categorías que emergen en contextos diversos (Sedema, 2012a):

\section{a) Sociales}

Las áreas verdes urbanas son importantes para la calidad de vida de los habitantes de las ciudades.

Proyectos de creación y manejo de nuevas áreas verdes en sitios menos favorecidos.

Destinar ahorro generado a las áreas verdes de las zonas menos favorecidas. 


\section{b) Ambientales \\ [...] ecosistemas que forman las áreas verdes en el DF. \\ [...] servicios ambientales.}

c) Económicos

Ahorros significativos en el manejo de áreas verdes públicas.

Incentivos fiscales por conservación de áreas verdes [...] otorgar un beneficio fiscal a los contribuyentes que conserven y den mantenimiento a las áreas verdes de su propiedad.

Reducción fiscal [cursivas nuestras].

Los temas que emergen de este análisis tienen una asociación con las categorías movilizadas en el ámbito internacional. La calidad de vida como guía de la política pública es un tema fundamental que considera a las áreas verdes urbanas desde un antropocentrismo ambiguo, aspectos ampliamente discutidos por autores como Amartya Sen y Martha Nussbaum (2002). Por ejemplo, en el Libro Blanco Reverdece tu ciudad y gestión de áreas verdes se presenta el término en la justificación del texto:

En los últimos años, la necesidad de incrementar y mantener en buen estado las áreas verdes urbanas ha cobrado una gran relevancia en las ciudades, ya que la calidad de vida de sus habitantes ha disminuido; las áreas verdes públicas en el Distrito Federal requieren de manejo y mantenimiento constantes, lo que significa una erogación de recursos que, en la mayoría de los casos, son insuficientes [Sedema, 2012a, p. 47; cursivas nuestras].

Además de observar la presencia de la categoría de calidad de vida vinculada al concepto de áreas verdes urbanas, de manera implícita se encuentran los lenguajes de la biología (al referirse al manejo y el mantenimiento) y de la economía (cuando se subraya la insuficiencia de recursos para realizar acciones públicas a favor de las áreas verdes).

Por otra parte, al referirse a los menos favorecidos, se atiende a una narrativa recurrente en las políticas públicas, aunque no tiene un marco teórico que le brinde la cohesión necesaria para considerarla una categoría como tal. Sin embargo, es importante señalar dicha narrativa porque frecuentemente las áreas verdes urbanas se presentan como solución al tema de la desigualdad urbana. Resulta de interés el hecho de que las políticas insistan en centrar la atención en las áreas verdes urbanas para resolver problemas de desigualdad que se vinculan con mayor fuerza a otro tipo de características y dinámicas socioeconómicas, más que al manejo de las áreas verdes. En este sentido, se puede decir que las áreas verdes urbanas, en cuanto al 
tema de la desigualdad, podrían considerarse como un distractor, puesto que es más fácil rehabilitar o manejar espacios verdes que conseguir la equidad en términos sociales dentro del contexto urbano.

En el documento que aquí se analiza, las categorías utilizadas en el discurso ambiental hacen referencia a una visión sistémica del medio ambiente, y en el centro del sistema, al ser humano que recibe los servicios ambientales generados en dichos espacios.

En lo económico, los programas aquí abordados son relevantes dada su vinculación con categorías como la de incentivos fiscales, entre los que se incluye la reducción fiscal, la cual podría ser considerada un concepto puesto que se refiere a una acción con mayor nivel de concreción que la relativa a los incentivos. Por otra parte, se busca un ahorro en el manejo de áreas verdes públicas, con la finalidad de reorientarlo hacia el cuidado de las áreas verdes ubicadas en las zonas menos favorecidas; en este sentido, el principio de equidad no sólo se piensa en términos de distribución de áreas verdes, sino de distribución de los recursos para el manejo de dichos espacios.

En cuanto a lo ambiental, se presentan dos categorías: ecosistemas y servicios ambientales. Esto resulta relevante dado que en los documentos de política ambiental la categoría de equilibrio ecológico, que considera una visión ecosistémica, suele presentarse después de la categoría de sustentabilidad. Sin embargo, en el Libro Blanco analizado, la visión ecosistémica pareciera ser un puente discursivo para la emergencia de los servicios ambientales a partir de la sustentabilidad, redirigiendo el énfasis hacia los aspectos socioculturales y económicos del medio ambiente, con una fuerza antropocéntrica mayor que cuando se hace referencia al equilibrio ecológico (visión ecosistémica), el cual no llega a constituirse nunca como el punto de llegada de la política ambiental.

En el Libro Blanco Reverdece tu ciudad y gestión de áreas verdes se hace referencia al Libro Blanco del Programa de Certificación para Edificaciones Sustentables (PCES), en donde la categoría que tiene más reiteraciones es la de sustentabilidad con diversas asociaciones (Ballinas, 2018). Sin embargo, esta misma categoría no tiene semejante relevancia en el Libro Blanco Reverdece tu ciudad y gestión de áreas verdes, puesto que la categoría sustentabilidad sólo se vincula a los espacios verdes cuando se hace alusión a las azoteas, pero no cuando se refiere a las áreas verdes a nivel de suelo: "con este sistema se crean azoteas verdes en edificaciones de diversos tipos, que generan beneficios ambientales y sociales para todos, lo cual ayuda a construir un México más sustentable con beneficio colectivo" (Sedema, 2012a, p. 42). 
La recurrencia de la categoría de calidad de vida y la nula presencia de la categoría sustentabilidad al referirse a las áreas verdes a nivel de suelo en el Libro Blanco Reverdece tu ciudad y gestión de áreas verdes, permite apreciar cómo ciertas categorías se posicionan como objetivos de la política pública y, al mismo tiempo, como criterios para su evaluación. Los ejemplos previos muestran la forma en que los conceptos, categorías y narrativas brindan continuidad al discurso de la política pública, puesto que se encuentran presentes en todas sus etapas y fluyen de una a otra sin encontrar límites y con posibilidades de seguir muchas direcciones, nunca como procesos lineales.

\section{Las narrativas recurrentes en las políticas de áreas verdes urbanas}

En el Libro Blanco Reverdece tu ciudad y gestión de áreas verdes están presentes narrativas que consideran al tiempo como un aspecto vinculado al discurso, en donde es posible reconocer algunos elementos para la comprensión del concepto de áreas verdes urbanas (Sedema, 2012a):

1) El tiempo para obtener la información sobre áreas verdes.

2) El tiempo para la creación del área verde.

3) El tiempo para resolver la problemática.

4) El tiempo para la planeación.

5) El tiempo para el manejo integral.

6) El tiempo de la jornada de intervención.

En la política de áreas verdes urbanas es importante considerar el tiempo de las áreas verdes y el tiempo de las estrategias que se proponen para ellas. Un reto es hacer coincidir ambos tiempos, aunque sea de relevancia, puesto que es en el tiempo y en el espacio donde se juega la práctica concreta de una política pública, con sus repercusiones.

En cuanto a la comprensión del espacio, se puede observar mediante contrastes una comprensión continua entre dos formas de entender lo que sucede en términos espaciales con las áreas verdes urbanas dentro del discurso de la política pública: a) entre el conocimiento y la acción, puesto que, en el discurso, el conocimiento emerge como causa (o fundamentación) de la acción; b) entre lo ambiental y las áreas verdes, cuya relación se presenta para vincular un ámbito de mayor amplitud (medio ambiente) en el que se incluye otro de menor amplitud (áreas verdes), el cual se propone como central puesto que se vincula a otros aspectos ambientales; $c$ ) entre lo 
urbano y las áreas verdes, pues se presenta una relación inclusiva, aunque la inclusión de las áreas verdes en el tema urbano pudiera ser periférica, si comparamos su importancia con la que tendría el tema de transporte y vivienda, por poner dos ejemplos; y $d$ ) entre la población y las áreas verdes, ya que se ofrece una interacción entre seres vivos que forman parte de un ecosistema.

La relación entre el conocimiento y la acción pública aparece como una dependencia, puesto que, si desde la acción pública no se toman las medidas necesarias para atender el problema ambiental, es porque no se cuenta con los conocimientos necesarios sobre dicho problema. Ello se convierte en una justificación de la acción pública a partir de la falta de conocimientos generados desde la ciencia y la tecnología. Sin embargo, al mencionar los conocimientos de las comunidades, se señala la importancia de construir una cultura del uso y cuidado de las áreas verdes urbanas. Esta acción, realizada por la ciudadanía, parece más factible que la acción gubernamental.

Cuando se alude a las áreas verdes relacionadas al ámbito urbano, no sólo se hace referencia a la recuperación, sino también a la compensación de dichas áreas en el interior del medio urbano. La naturación se constituye entonces como una estrategia para resarcir el sentimiento de culpabilidad social ante el deterioro ambiental; por tal motivo, se convierte en una estrategia que obtiene fuerza a partir del entendimiento ético de la compensación.

Por otro lado, la interacción entre población y áreas verdes es un ámbito que permite integrar la participación ciudadana con relativa facilidad, puesto que no trastoca directamente el orden social y político, aunque pueda tener fines políticos. Sin embargo, participar en acciones puntuales de cuidado de las áreas verdes brinda a los participantes la sensación de control, aun cuando ese control tenga límites muy estrechos, si se piensa en la complejidad de la problemática ambiental, social y económica que se relaciona con la sustentabilidad.

En cuanto al vínculo entre lo ambiental y las áreas verdes, destaca el papel relevante que en el Libro Blanco analizado se les otorga a las actividades educativas relacionadas con la construcción de una cultura ambiental en donde, por ejemplo, la presencia de jardineros y de ciudadanos es considerada un factor que incide sobre la acción pública destinada al mejoramiento del ambiente urbano.

Finalmente, se puede afirmar que los discursos de las políticas de áreas verdes urbanas tienen componentes temporales y espaciales que se tendrían que considerar para identificar las dinámicas narrativas que permiten la construcción de diversas comprensiones de la acción pública, en las cuales también se incluye al componente poblacional. 


\section{Conclusiones}

La comprensión de un concepto como el de áreas verdes urbanas, en el ámbito de las políticas públicas, demanda su observación bajo distintas aristas, de modo que pueda ser ubicado en términos discursivos con significaciones más complejas que las que pueden encontrarse en una definición. De esta forma, es posible entender la relevancia que tiene el concepto en relación consigo mismo, con otros conceptos y con las diversas categorías que se movilizan en el discurso ambiental.

El análisis discursivo desarrollado en este trabajo permite apreciar las similitudes, las diferencias, e incluso las contradicciones respecto a las significaciones que se atribuyen al concepto central que es objeto de estudio (áreas verdes urbanas). La confluencia de múltiples definiciones, funciones y significados permite reconocer a las áreas verdes urbanas como un concepto polisémico, cuyos significados entrelazan aspectos sociales, culturales, económicos y ambientales, entre otros.

Por otra parte, los conceptos utilizados en las políticas públicas se movilizan en un espacio discursivo en el que convergen lenguajes disciplinares y en donde se entrelazan diversas escalas que se anidan en programas propuestos para tiempos y espacios específicos, siendo el territorio una expresión de ello. La forma en que el lenguaje económico se introduce en las políticas ambientales conduce a la afirmación de que no es posible realizar un análisis de políticas públicas sin la consideración de actores sociales relacionados con las instituciones financieras, dada su influencia en la construcción discursiva de la política ambiental mediante la movilización de conceptos, categorías y narrativas (Spash y Aslaksen, 2015).

El lenguaje biológico, a pesar de ser el más evidente y extenso, cumple sólo una función instrumental, ya que el conocimiento científico al que se refiere difícilmente puede ser incorporado a las acciones públicas debido al problema mencionado en el mismo documento: la falta de planeación de las áreas verdes en el ámbito de lo urbano.

Finalmente, el lenguaje normativo se mantiene como una posibilidad para plasmar aquellas prioridades establecidas por la gestión pública local y establecer criterios para actuaciones futuras.

A partir del análisis desarrollado es posible concluir que, para el caso de la Ciudad de México, la construcción de las políticas de áreas verdes urbanas durante el periodo analizado se llevó a cabo más desde los intereses económicos que desde la comprensión de dichos espacios desde una perspectiva ecosistémica, aun cuando el discurso insista en las ventajas ambientales y sociales de su manejo y conservación. 
En cuanto a las narrativas, ha sido interesante identificar los puentes discursivos que relacionan el concepto central con otros conceptos y categorías con disposiciones discursivas específicas, que van a utilizar el tema de las áreas verdes urbanas para fines más amplios en el sentido temático o distinto de lo ambiental.

En el caso analizado, por ejemplo, resulta interesante constatar la ausencia de una vinculación discursiva entre las nociones de sustentabilidad y las áreas verdes urbanas a nivel de suelo, y, en contraparte, encontrar una relación estrecha entre dicha categoría y las azoteas verdes. En este sentido, pareciera que durante el período gubernamental analizado existió mayor interés político en las acciones vinculadas a la naturación de azoteas -mismas que ameritaron, incluso, la creación de un programa específico, con su propio Libro Blanco-, que en las acciones orientadas a la gestión de áreas verdes a nivel de suelo.

El ejercicio desarrollado a lo largo de este trabajo evidencia al discurso como un espacio de gran relevancia en los procesos de legitimación de las políticas públicas. El estudio de los procesos discursivos permite mantener cierta libertad frente a lo que se nos muestra como "verdades unívocas" y asumir una posición crítica frente a la construcción de problemas, soluciones, situaciones, u oportunidades que se nos presentan como "únicas" desde las políticas públicas.

\section{Bibliografía}

Aguilar, L. F. (2003). Estudio Introductorio. En L. F. Aguilar Villanueva (ed.), La hechura de las políticas públicas (pp. 15-84). Ciudad de México: Miguel Ángel Porrúa / Librero Editor.

Ballinas, M. L. (2018). Análisis del proceso discursivo de la política de áreas verdes urbanas en la Ciudad de México (2006-2012) (Tesis doctoral, El Colegio de México, A.C., Ciudad de México). Recuperado de https:// colmex.userservices.exlibrisgroup.com/view/delivery/52COLMEX INST/1282392110002716

Bourdieu, P. (2008). ¿Qué significa hablar? Economía de los intercambios lingüísticos. España: Akal.

Centenera, F. (2015). La claridad legislativa en el pensamiento de Lon L. Fuller: un análisis desde la teoría de la legislación. Anales del Derecho, 33(1), 1-27. Recuperado de https://revistas.um.es/analesderecho/ article/view/188801/180361 
Conde, G. (2017). Agua, poder y hegemonía entre actores estatales y no estatales en Turquía, Siria e Iraq. Estudios de Asia y África, 52(1), 5-28. Recuperado de https://estudiosdeasiayafrica.colmex.mx/index.php/eaa/ article/view/2226/2230

Conferencia de Naciones Unidas sobre el Medio Ambiente y el Desarrollo. (1992). Cumbre de la tierra de Río de Janeiro. Recuperado de https:// www.un.org/spanish/esa/sustdev/documents/declaracionrio.htm

Durand, L. (2014). ¿Todos ganan? Neoliberalismo, naturaleza y conservación en México. Sociológica, 29(82), 183-223. Recuperado de http:// www.scielo.org.mx/pdf/soc/v29n82/v29n82a6.pdf

Fairclough, N. (2008). El análisis crítico del discurso y la mercantilización del discurso público: las universidades. Discurso y Sociedad, 2(1), 170-185. Recuperado de http://www.dissoc.org/ediciones/v02n01/DS2 (1)Fairclough.pdf

Gellers, J. C. (2015). Greening critical discourse analysis. Applications to the study of environmental law. Critical Discourse Studies, 12(4), 482493. Recuperado de https://www.tandfonline.com/doi/full/10.1080/17 405904.2015.1023326

Illich, I. (1990). Toward a history of needs. Nueva York, NY: Random House. Koselleck, R. (2003). Futuro-pasado. Para una semántica de los tiempos históricos. España: Paidós.

Ley Ambiental del Distrito Federal. (2000). Gaceta Oficial del Distrito Federal. México, 13 de enero (última reforma: GODF, 3-05-2011).

Ley General del Equilibrio Ecológico y Protección al Medio Ambiente. (1988). Diario Oficial de la Federación. México, 28 de enero (última reforma: $D O F, 16-01-2014)$.

March, H. (2013). Neoliberalismo y medio ambiente: una aproximación desde la geografía crítica. Documents d'Anàlisi Geográfica, 59(1), 137-153. Recuperado de https://core.ac.uk/download/pdf/39021279. pdf

Molle, F. (2008). Nirvana concepts, narratives and policy models: Insight from the water sector. Water Alternatives, 1(1), 131-156. Recuperado de http://www.water-alternatives.org/index.php/allabs/20-a-1-1-8/file

Mühlhäusler, P. y Peace, A. (2006). Environmental discourses. Annual Review of Anthropology, 35, 457-479. Recuperado de https://www.annual reviews.org/doi/full/10.1146/annurev.anthro.35.081705.123203

Nusbaum, M. C. y Sen, A. (2002). La calidad de vida. Ciudad de México: Fondo de Cultura Económica.

Rente, J., Krishnamurthy, L. y Keipi, K. J. (1998). Áreas verdes urbanas en América Latina: una introducción. En L. Krishnamurthy y J. Rente 
(eds.), Áreas verdes urbanas en Latinoamérica y el Caribe (pp. 1-13). México: Universidad Autónoma de Chapingo.

Secretaría de Medio Ambiente del Distrito Federal (Sedema). (2012a). Reverdece tu ciudad y gestión de áreas verdes urbanas. Ciudad de México: Sedema (Libro Blanco).

Secretaría de Medio Ambiente del Distrito Federal (Sedema). (2012b). Programa de Certificación de Edificaciones Sustentables. Ciudad de México: Sedema (Libro Blanco).

Spash, C. L. y Aslaksen, L. (2015). Re-establishing an ecological discourse in the policy debate over how to value ecosystems and biodiversity. Journal of Environmental Management, 159, 245-253. Recuperado de https:// www.sciencedirect.com/science/article/pii/S0301479715300384

Van Dijk, T. (1991). Estructuras y funciones del discurso. Ciudad de México: Siglo XXI.

\section{Acerca de los autores}

María Luisa Ballinas Aquino es doctora en Estudios Urbanos y Ambientales por El Colegio de México, A.C.; maestra en Ciencias en Recursos Naturales y Desarrollo Rural por El Colegio de la Frontera Sur; licenciada en Ciencias Religiosas por la Universidad La Salle; y química farmacéutica bióloga por la Universidad Veracruzana. Sus líneas de investigación son: significados de la calidad de vida, religión y medio ambiente; gestión de residuos sólidos en el ámbito escolar; estudios socioambientales en cuencas hidrológicas; políticas y gestión de áreas verdes; análisis del discurso de las políticas públicas. Pertenece a la Red de Investigadores del Fenómeno Religioso en México, a la Asociación Latinoamericana de Estudios del Discurso, y al Sistema Estatal de Investigadores en Chiapas. Recibió el premio de la Fundación BBVA-Bancomer a la excelencia Colmex en 2013 y 2014. ORCID: https://orcid.org/0000-0002-7381-2363

José Álvaro Hernández Flores es doctor en Ciencias con especialidad en Estrategias para el Desarrollo Agrícola Regional por el Colegio de Postgraduados, y licenciado en Economía por la Universidad Nacional Autónoma de México. Es miembro del Sistema Nacional de Investigadores, nivel I. Ha desarrollado estancias de investigación en el Instituto Gino Germani de la Universidad de Buenos Aires y en el Centro de Estudios del Desarrollo Económico y Social (CEDES) de la Benemérita Universidad Autónoma de Puebla. Actualmente se encuentra adscrito como catedrático Conacyt al Centro 
de Estudios Demográficos, Urbanos y Ambientales de El Colegio de México, A.C. Entre sus líneas de investigación destacan: desarrollo sustentable, estrategias de reproducción social, y configuración de los territorios rurales y periurbanos. ORCID: http://orcid.org/0000-0002-8349-0505

Recepción: 22 de marzo de 2019. Aceptación: 17 de junio de 2019. 